\title{
Chitinase-3-like Protein 1 (YKL-40): A New Biomarker of Inflamma- tion in Pyoderma Gangrenosum
}

\author{
Alina JANKOWSKA-KONSUR ${ }^{1}$, Magdalena $Ł Y K{ }^{1,2}$, Klaudia RUBAS ${ }^{1}$, Danuta NOWICKA-SUSZKO ${ }^{1}$, Joanna MAJ ${ }^{1}$ and Jacek \\ C. SZEPIETOWSKI ${ }^{1}$ \\ ${ }^{1}$ Department of Dermatology, Venereology and Allergology, Wroclaw Medical University, Wroclaw, and ${ }^{2}$ Student Research Group of Experimental \\ Dermatology, Department of Dermatology, Venereology and Allergology, Wroclaw Medical University, Wroclaw, Poland
}

\begin{abstract}
Pyoderma gangrenosum (PG) is a rare, neutrophilic dermatosis with unclear aetiopathology, considered as an autoinflammatory disease, associated with other immune-mediated disorders. Chitinase-3-like protein 1 (YKL-40) is an inflammatory biomarker secreted by a wide variety of cells, including neutrophils. To evaluate YKL-40 serum level in relation to clinicopathological data, 48 patients with PG and 40 healthy controls were enrolled in the study. Skin lesions were measured to calculate the affected area. Inflammatory parameters (C-reactive protein, white blood cell count with neutrophils) were determined from blood samples. YKL-40 and IL-6 levels were measured in serum by enzyme-linked immunosorbent assay. YKL-40 serum level was significantly higher in patients with PG than in controls (58.4 vs $36.4 \mathrm{ng} / \mathrm{ml}$, respectively; $p<0.00001)$. The positive correlation between YKL-40 level and IL-6 level was observed $(r=0.48, p=0.0006)$ along with a trend towards significance of relationship between YKL-40 level and C-reactive protein ( $r=0.28$, $p=0.052$ ). YKL-40 can be considered a valuable biomarker of inflammation in PG.
\end{abstract}

Key words: pyoderma gangrenosum; chitinase-3-like protein 1; YKL-40; biomarker; inflammation.

Accepted Dec 22, 2021; Epub ahead of print Dec 22, 2021

Acta Derm Venereol 2022; 102: adv00646.

DOI: $10.2340 /$ actadv.v101.978

Corr: Jacek C. Szepietowski, Department of Dermatology, Venereology and Allergology, Wroclaw Medical University, PL-50-368 Wrocław, Poland. E-mail: jacek.szepietowski@umed.wroc.pl

$\mathrm{P}$ yoderma gangrenosum (PG) is a rare neutrophilmediated dermatosis (ND) with an estimated incidence of 3-10 cases per million population per year (1). The pathogenesis of the disease is not fully understood; however, altered neutrophil trafficking and activation, as well as genetic factors and systemic inflammation, are considered $(2,3)$. Due to characteristic phenomena, such as recurrent sterile tissue inflammation, without circulating autoantibodies and autoreactive T-cells, PG has recently been recognized as belonging to the spectrum of autoinflammatory disorders (4). It is also a part of classical autoinflammatory syndromes, i.e. pyogenic arthritis, PG, and acne (PAPA); and PG, acne, and hidradenitis suppurativa (PASH) (5). PG is frequently associated with different conditions, in particular inflam-

\section{SIGNIFICANCE}

Elevated chitinase-3-like protein 1 serum level has been reported in many inflammatory disorders; however, it has not yet been described in pyoderma gangrenosum. This study compared chitinase-3-like protein 1 serum level in patients with pyoderma gangrenosum and healthy controls, and evaluated chitinase-3-like protein 1 level in relation to clinicopathological data. The results suggest that chitinase-3-like protein 1 is one of the most sensitive biomarkers of inflammation in pyoderma gangrenosum, and is more valuable than commonly used inflammatory markers. These findings will serve as a basis for future studies comparing chitinase-3-like protein 1 serum level in other ulcerative dermatosis, and exploring the potential use of chitinase-3-like protein 1 to differentiate those conditions.

matory bowel diseases and rheumatological diseases, and haematological disorders, with which it shares the features of impaired immune system functions (6).

Chitinase-3-like protein 1 (CHI3L1,) also known in humans as YKL-40, is a $40-\mathrm{KDa}$ chitin-binding lectin, member of the glycosyl hydrolase family 18 . Initially, it was discovered in the culture supernatant of osteosarcoma cell line MG63 and later detected in synovial cells and chondrocytes (7-9); however, it is also produced by a wide variety of cells, including neutrophils, macrophages, fibroblast-like cells, endothelial cells, smooth muscle cells and cancer cells (10-12). The synthesis and secretion of YKL-40 are regulated by growth factors, extracellular matrix changes, stress, cytokines, and drugs (13-16). Recent studies indicate a potential role of YKL-40 as a marker of inflammation, tissue remodelling, and fibrosis, proliferation and angiogenesis in a multitude of conditions, including inflammatory disorders, dermatoses, malignancies, cardiovascular diseases, and infections $(7,17-20)$.

To the best of our knowledge, this is the first study to assess the expression of YKL-40 in PG. The aim of the current study was to evaluate the serum level of YKL-40 in relation to clinicopathological data in PG.

\section{MATERIALS AND METHODS}

This case-control study included 48 patients with PG recruited between 2015 and 2020 in the Department of Dermatology, Venereology and Allergology of Wroclaw Medical University, Poland. 
All patients were referred to the department by wound specialists, dermatologists and surgeons from out-patient clinics in south-west Poland for diagnosis or verification of diagnosis. Upon admission to the clinic, all patients participating in the study were evaluated by 2 independent dermatologists and diagnosed with ulcerative PG and had inflammatory lesions. Patients were included in the study based on the achievement of 10 or more points in the PARACELSUS score (21). Patients with active comorbidities or receiving systemic treatment were excluded due to the possibility of the inference to study parameters. At admission, the number of lesions was assessed, and the area of skin lesions was measured and calculated. All included patients were negative for malignancies and had no clinical symptoms of infection. Table I presents a detailed description of studied PG patients.

The control group comprised 40 healthy volunteers, with no history of PG or any other autoinflammatory diseases.

Serum samples were collected from the patients at the admission for the assessment of the serum concentration of YKL-40, C-reactive protein (CRP), interleukin-6 (IL-6), white blood cell count (WBC), and neutrophil count (NEUT).

The samples were collected, stored and labelled according to the R\&D Systems ready-made enzyme-linked immunoassay (ELISA) kits procedure for human chitinase 3-like immunoassay. The serum separator tube (SST) was used. After collection and clotting, the blood was centrifuged at 4,500 rpm. Separated serum was frozen and stored regarding the manufacturers' instruction at the temperature of $-70^{\circ} \mathrm{C}$ until it was processed. The ELISA kits were used to quantify YKL-40 (Quantikine ${ }^{\circledR}$ ELISA Human Chitinase 3-like 1 Catalog No. DC3L10 Lot P230972) and IL-6 (Quantikine ${ }^{\circledR}$ HS ELISA Human IL-6 Catalog No. HS600C) Quantitative analysis of YKL-40 and IL- 6 concentrations were carried out using the Epoch spectrophotometer (BioTek Instruments, Winooski, VT, USA). Serum CRP levels were determined using the turbidimetric assay on ARCHITECT ${ }^{\circledR}$ ci4100 ${ }^{\mathrm{TM}}$ analyser (Abbott Diagnostics, Lake Forest, IL, USA). WBC and neutrophil counts were measured using fluorescent flow cytometry Sysmex XN-2000 ${ }^{\mathrm{TM}}$ analyser (Sysmex, Kobe, Japan).

The Shapiro-Wilk test was used to check the data distribution. All quantitative variables were described as median and interquartile range (IQR) or mean \pm standard deviation (SD). Comparisons between the groups were performed by the Mann-Whitney $U$ test. Correlations between the variables were calculated using
Table I. Clinical characteristics of the group of patients with pyoderma gangrenosum

\begin{tabular}{ll}
\hline Characteristics & Baseline value \\
\hline Age, years, mean \pm SD & $53.3 \pm 16.9$ \\
Male/female, $n(\%)$ & $17(35.4) / 31(64.6)$ \\
Area of skin lesions, $\mathrm{cm}^{2}$, median (IQR) & $19.6(64.135)$ \\
Localization of skin lesions, $n(\%)$ & \\
Face and neck & $1(2.1)$ \\
Upper extremity & $5(10.4)$ \\
Trunk & $8(16.7)$ \\
Lower extremity & $41(85.4)$ \\
Number of lesions, $n(\%)$ & \\
1 & $20(41.7)$ \\
2 & $16(33.3)$ \\
3 or more & $12(25.0)$ \\
Comorbidities, $n$ (\%) & $6(12.5)$ \\
Inflammatory bowel diseases & $4(8.3)$ \\
Rheumatoid arthritis/spondyloarthropathies & $2(4.2)$ \\
Systemic lupus erythematous/systemic sclerosis/ & \\
$\quad$ Sjögren syndrome & \\
\hline
\end{tabular}

SD: standard deviation; IQR: interquartile range.

Spearman's rank correlation. A receiver operating characteristic curve (ROC) was performed to identify the optimal cut-off value for patients-controls differentiation. The area under the curve (AUC) value was calculated to determine the accuracy of the test. $p$-value $<0.05$ was considered statistically significant. Statistical analysis was performed using Statistica 13.3 software (StatSoft Inc., Tulsa, OK, USA).

The study was conducted in compliance with ethical regulations and follows the principles of the Declaration of Helsinki. The study has been approved by the Bioethics Committee of the Wrocław Medical University. The patients in this manuscript provided written informed consent to publication of their case details.

\section{RESULTS}

Among 48 patients with PG, 31 (64.6\%) were women and $17(35.4 \%)$ were men aged from 19 to 85 years (mean \pm SD $53.3 \pm 16.9$ years). The control group comprised 40 volunteers, 21 (52.5\%) women and 19 (47.5\%) men, aged from 24 to 84 years (mean \pm SD $48.2 \pm 13.0$ ).
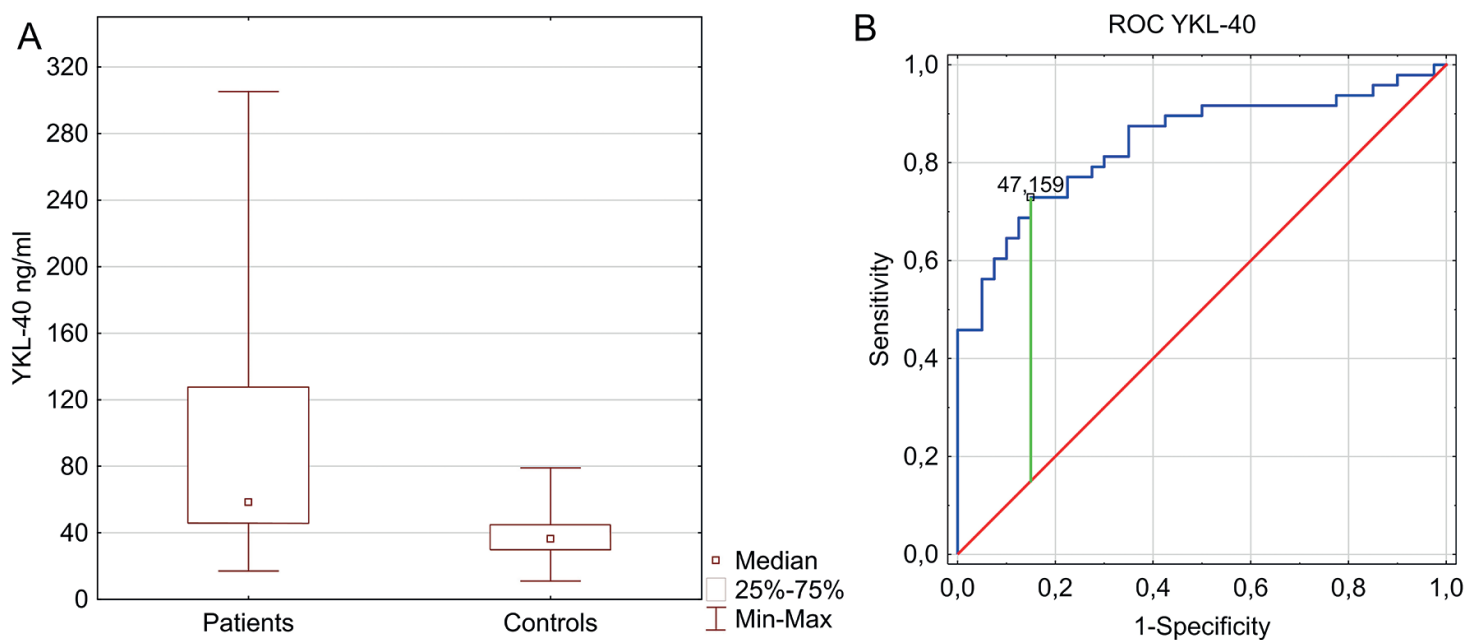

Fig. 1. Significant difference in the serum chitinase-3-like protein $\mathbf{1}$ (YKL-40) concentration in patients with pyoderma gangrenosum (PG) and the control group. (A) The median serum YKL-40 level in the group of patients with PG was significantly higher than in the control healthy subjects $(p<0.00001)$. (B) The receiver operating characteristic (ROC) curve presented the area under the curve of $0.841(95 \% \mathrm{CI}: 0.757-0.924, p<0.00001)$. Based on this value we assume that YKL-40 is a good test to differentiate between patients and healthy individuals. The optimal cut-off point for serum YKL-40 level was estimated at $47.159 \mathrm{ng} / \mathrm{ml}$. 

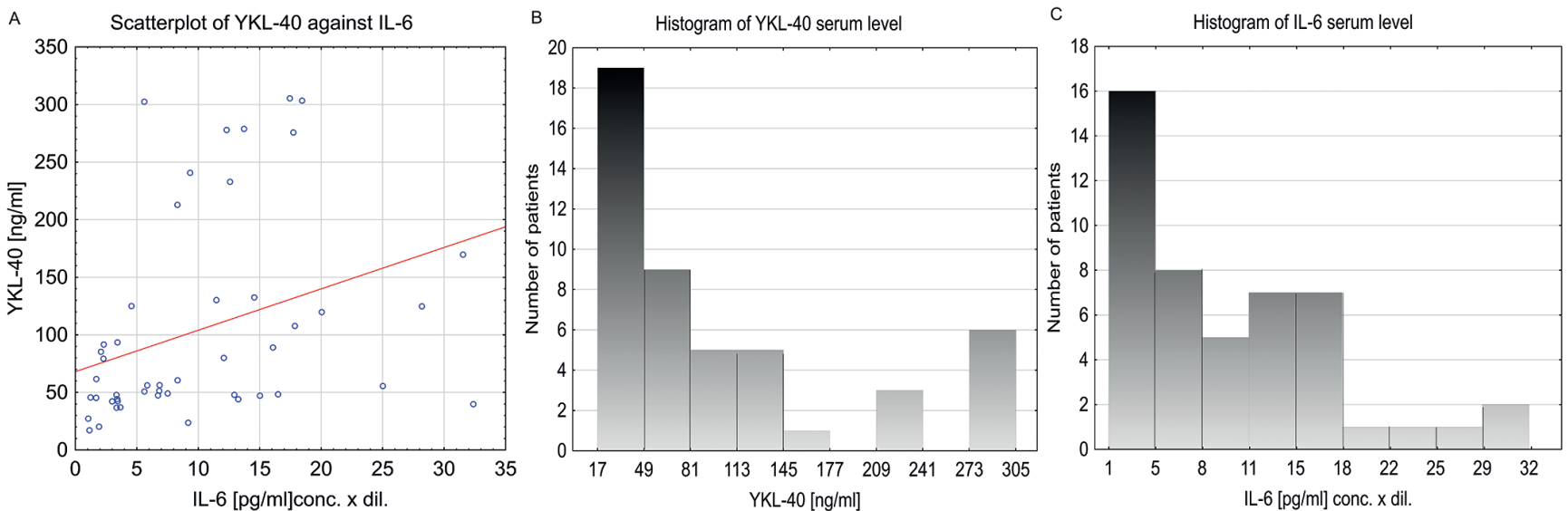

Fig. 2. Correlation between chitinase-3-like protein 1(YKL-40) and interleukin (IL)-6 concentrations in patients with pyoderma gangrenosum. (A) Scatterplot of YKL-40 concentration against IL-6 concentration. (B) Histogram showing distribution of YKL-40 serum level. (C) Histogram showing distribution of IL-6 serum level.

The median serum YKL-40 level in the group of patients with $\mathrm{PG}$ was significantly higher than in the control healthy subjects (58.4(IQR 80.7$) \mathrm{ng} / \mathrm{ml}$ and 36.4 (IQR 14.7) $\mathrm{ng} / \mathrm{ml}$, respectively; $(p<0.00001)$ (Fig. 1).

The optimal cut-off point for serum YKL-40 level was $47.159 \mathrm{ng} / \mathrm{ml}$. The sensitivity and specificity of serum YKL-40 for the diagnosis of PG were $73 \%$ and $85 \%$, respectively. ROC analysis presented the area under the curve of 0.841 (95\% CI: $0.757-0.924, p<0.00001$ ), with high negative (NPV) and positive (PPV) predictive values of 0.71 and 0.85 , respectively (Fig. 1).

Spearman's rank correlation test revealed a significant positive relationship between serum YKL-40 level and IL-6 level ( $r=0.48, p=0.0006)$. (Fig. 2) No significant correlations were found between serum YKL-40 levels and other laboratory parameters, such as WBC or NEUT; however, a distinct trend towards significance between YKL-40 and CRP was observed ( $p=0.052)$ (Table II). The median serum CRP level was $8.7 \mathrm{mg} / \mathrm{l}$ and was elevated in 28 patients $(58.3 \%)$. The median value of WBC was $7.8 \times 10^{9} / 1$ and was elevated in $18(37.5 \%)$ patients. Detailed laboratory findings in patients with $\mathrm{PG}$ are shown in Table III. In the control group, the median IL-6 serum level was 0.982 (IQR 0.525) pg/ml.

No significant correlations were found between serum YKL-40 levels and age, area of skin lesions, and number of skin lesions (Table II). Sex, smoking, and pathergy

Table II. Correlation analysis for chitinase-3-like protein 1 and other clinical data in patients with pyoderma gangrenosum

\begin{tabular}{llcl}
\hline Variables & $n$ & $\begin{array}{l}\text { Spearman rank correlation } \\
\text { coefficient }(r)\end{array}$ & $p$-value \\
\hline CRP & 48 & 0.28 & 0.05 \\
WBC & 48 & 0.10 & 0.48 \\
NEUT & 14 & -0.281 & 0.33 \\
IL-6 & 48 & 0.48 & $\mathbf{0 . 0 0 0 6}$ \\
Area of skin lesions & 48 & 0.04 & 0.79 \\
Number of skin lesions & 48 & 0.04 & 0.80 \\
\hline
\end{tabular}

CRP: C-reactive protein; WBC: white blood cell count; NEUT: neutrophil count; IL: interleukin. A significant positive relationship between serum YKL-40 level and IL-6 level is marked in bold phenomenon were not found to be determining factors for YKL-40 serum concentration (detailed data not shown). Lower YKL-40 level was observed in patients with lesions located on the trunk compared with other regions, although the difference did not reach statistical significance $(p=0.058)$.

A history of comorbidities, such as inflammatory bowel diseases (IBD) $(p=0.89)$, rheumatoid arthritis $(p=0.61)$, and systemic rheumatoid diseases $(p=0.70)$, did not affect the YKL-40 level.

\section{DISCUSSION}

This study is the first to show that the serum concentration of YKL-40 in patients with PG is significantly elevated compared with healthy individuals. These results may indicate a role of YKL-40 in the pathomechanisms of PG, considering that the level of YKL-40 was determined in the group of patients with PG without active comorbidities. The possible impact of other diseases on YKL-40 levels cannot be excluded. This is a limitation of the study.

In recent years, elevated YKL-40 level has been reported in a wide range of diseases and pathological processes, including inflammatory disorders that remain in close association with PG. Several studies have reported elevated serum levels of YKL-40 in patients with IBD; however, the interpretations of these results is contra-

Table III. Laboratory findings in patients with pyoderma gangrenosum

\begin{tabular}{lccc}
\hline Laboratory parameter & Min-Max & Mean \pm SD & Median (IQR) \\
\hline YKL-40 $(\mathrm{ng} / \mathrm{ml})$ & $17.1-305.3$ & $104.2 \pm 87.9$ & $58.4(80.7)$ \\
CRP $(\mathrm{mg} / \mathrm{l})$ & $0.3-64.4$ & $14.9 \pm 16.7$ & $8.7(18.7)$ \\
WBC $\left(\times 10^{9} / \mathrm{l}\right)$ & $3.9-19.9$ & $9.3 \pm 3.6$ & $7.8(4.4)$ \\
NEUT $\left(\times 10^{9} / \mathrm{l}\right)$ & $2.0-17.0$ & $6.0 \pm 4.1$ & $4.9(2.9)$ \\
IL-6 $(\mathrm{pg} / \mathrm{ml})$ & $1.1-32.4$ & $10.1 \pm 8.1$ & $7.9(11.3)$ \\
\hline
\end{tabular}

YKL-40: chitinase-3-like protein 1; CRP: C-reactive protein; WBC: white blood cell count; NEUT: neutrophil count; IL: interleukin; Min: minimum; max: maximum; SD: standard deviation; IQR: interquartile range. 
dictory (22-24). Koutroubakis et al. (22) observed an association between the increased level of YKL-40 and disease activity, but found no correlation with fibrosis processes in the course of IBD. In contrast, a study by Erzin et al. (23) showed a statistically significant relationship, not only with disease activity, but also with the presence of strictures in Crohn's disease. Another report showed a statistically higher concentration of YKL-40 in the serum of patients with IBD and arthritis than in the group without arthritis (24). A few studies focused on the YKL-40 function in rheumatoid arthritis (RA), suggesting a role in the pathogenesis of RA and disease activity (25-27). YKL-40 was reported to be a useful predictor of joint inflammation and cartilage destruction (28, 29). On the other hand, the studied marker serum level was not predictive of clinical remission or radiographic progression in rheumatoid arthritis (30).

To date, several autoinflammatory syndromes have been identified, including: pyoderma gangrenosum, acne, and pyogenic arthritis (PAPA); pyoderma gangrenosum, acne, and hidradenitis suppurativa (PASH); pyoderma gangrenosum, acne, pyogenic arthritis, and hidradenitis suppurativa (PAPASH); psoriatic arthritis, pyoderma gangrenosum, acne, and hidradenitis suppurativa (PsAPASH); pyoderma gangrenosum, acne, and spondyloarthritis (PASS); and pyoderma gangrenosum, acne, and ulcerative colitis (PAC). Worldwide, studies on the role of YKL40 in autoinflammatory syndromes are lacking, but there are a few reports concerning the YKL-40 function in some diseases included in the syndromes in question.

Increased expression in the lesional skin and elevated serum concentration of YKL-40 was observed in hidradenitis suppurativa (HS) $(31,32)$. The YKL-40 serum level correlated significantly with the disease severity regarding the Hurley stage and reflected the degree of inflammation (32).

The elevated YKL-40 serum concentration was also reported in patients with acne vulgaris (33). In the quoted study, no association was found between the YKL-40 level and the severity of the disease, but a positive correlation was observed between the concentration of the discussed factor and total cholesterol, triglycerides, and low-density lipoprotein (LDL) and negative correlation with high-density lipoprotein (HDL), which may indicate other mechanisms leading to the increase in YKL-40 level.

In several studies on psoriatic arthritis, it has been postulated that YKL-40 may be a marker of disease activity serving as a useful tool in the diagnostic process and monitoring the response to therapy $(34,35)$.

Numerous research studies point to YKL-40 as a marker of inflammation. In the current study YKL-40 concentration was correlated with proinflammatory cytokine IL-6. We also observed a trend towards signifi- cance between YKL-40 and CRP. A positive correlation between IL- 6 and YKL-40 serum concentration was documented in Behçet's disease and Sjögren syndrome $(36,37)$. IL-6 plays a role in the regulation of YKL-40 levels during inflammation and the stimulatory effect of IL- 6 on the YKL-40 synthesis and serum concentration was documented in the study by Nielsen et al. (38).

Correlation between YKL-40 and CRP was reported in HS and familial Mediterranean fever (32). Similar results were presented in the study of Vind et al. (39), who demonstrated correlation between the studied marker with inflammatory parameters, such as CRP, WBC, and serum albumin in IBD.

According to the results of the current study, YKL-40 appears to be a more sensitive inflammatory marker than other well-known markers, including CRP, WBC, and neutrophil count. Similar conclusions were reached by Salomon et al. (40), who demonstrated that YKL-40 is a more sensitive marker than CRP and neutrophil count regarding inflammation degree in psoriasis vulgaris. The observed differences may indicate that YKL-40 represents different aspects of inflammation than other mediators.

Taking into account observed lack of correlation between the level of YKL-40 and wound size in patients with PG, it can be assumed that the increased level of YKL-40 reflects the general inflammatory process rather than the local activity of the disease. Similarly, in psoriasis vulgaris, no association between YKL-40 serum concentration and severity of the skin changes was observed (40). Considering the possible role of YKL-40, not only in inflammatory processes, but also in wound healing and fibrosis, elevated levels of YKL-40 could be expected in cutaneous ulcers of a different aetiology, including normal acute wounds. Therefore, future studies comparing YKL-40 serum levels in ulcerative dermatoses are needed, since they could provide new interesting data about the potential use of YKL-40 to differentiate those conditions.

In conclusion, this is the first report to document increased YKL-40 serum concentration in PG. The results indicate that YKL-40 could serve as a potential biomarker of inflammation in PG, which may be more sensitive than other classical, commonly used markers of inflammation. YKL-40 may help identify patients with increased systemic inflammation. This knowledge may be useful in making therapeutic decisions, as these patients may require intensification of standard treatment; however, this is the first study on the role of YKL-40 in PG, and further, more detailed, research is needed on this issue.

\section{ACKNOWLEDGEMENTS}

The study was supported by Reserach Grant from Wroclaw Medical University (SUBZ.C260.22.056).

The authors have no conflicts of interest to declare. 


\section{REFERENCES}

1. Ruocco E, Sangiuliano S, Gravina A, Miranda A, Nicoletti G. Pyoderma gangrenosum: an updated review. J Eur Acad Dermatology Venereol 2009; 23: 1008-1017.

2. Ahronowitz I, Harp J, Shinkai K. Etiology and management of pyoderma gangrenosum: A comprehensive review. Am J Clin Dermatol 2012; 13: 191-211.

3. Adachi $Y$, Kindzelskii AL, Cookingham G, Shaya S, Moore $\mathrm{EC}$, Todd 3rd RF, et al. Aberrant neutrophil trafficking and metabolic oscillations in severe pyoderma gangrenesum. J Invest Dermatol 1998; 111: 259-268.

4. Satoh TK, Mellett M, Contassot E, French LE. Are neutrophilic dermatoses autoinflammatory disorders? $\mathrm{Br} J$ Dermatol 2018; 178: 603-613.

5. Marzano AV, Ortega-Loayza AG, Heath M, Morse D, Genovese G, Cugno M. Mechanisms of inflammation in neutrophilmediated skin diseases. Front Immunol 2019; 10: 1059.

6. Ashchyan HJ, Nelson CA, Stephen S, James WD, Micheletti RG, Rosenbach M. Neutrophilic dermatoses: pyoderma gangrenosum and other bowel- and arthritis-associated neutrophilic dermatoses. J Am Acad Dermatol 2018; 79: 1009-1022.

7. Johansen JS. Studies on serum YKL-40 as a biomarker in diseases with inflammation, tissue remodelling, fibroses and cancer. Dan Med Bull 2006; 53: 172-209.

8. Hakala BE, White C, Recklies AD. Human cartilage gp-39, a major secretory product of articular chondrocytes and synovial cells, is a mammalian member of a chitinase protein family. J Biol Chem 1993; 268: 25803-25810.

9. Shackelton LM, Mann DM, Millis AJ. Identification of a 38kDa heparin-binding glycoprotein (gp38k) in differentiating vascular smooth muscle cells as a member of a group of proteins associated with tissue remodeling. J Biol Chem 1995; 270: 13076-13083.

10. Volck B, Price PA, Johansen JS, Sørensen O, Benfield TL, Nielsen $\mathrm{HJ}$, et al. YKL-40, a mammalian member of the chitinase family, is a matrix protein of specific granules in human neutrophils. Proc Assoc Am Physicians 1998; 110: 351-360.

11. Renkema GH, Boot RG, Au FL, Donker-Koopman WE, Strijland A, Muijsers AO, et al. Chitotriosidase a chitinase, and the 39-kDa human cartilage glycoprotein, a chitin-binding lectin, are homologues of family 18 glycosyl hydrolases secreted by human macrophages. Eur J Biochem 1998; 251: 504-509.

12. Zhao T, Su Z, Li Y, Zhang X, You Q. Chitinase-3 like-protein-1 function and its role in diseases. Signal Transduct Target Ther 2020; 5: 201

13. Johansen JS, Olee T, Price PA, Hashimoto S, Ochs RL, Lotz M. Regulation of YKL-40 production by human articular chondrocytes. Arthritis Rheum 2001; 44: 826-837.

14. Kzhyshkowska J, Mamidi S, Gratchev A, Kremmer E, Schmuttermaier C, Krusell L, et al. Novel stabilin-1 interacting chitinase-like protein (SI-CLP) is up-regulated in alternatively activated macrophages and secreted via lysosomal pathway. Blood 2006; 107: 3221-3228.

15. De Ceuninck F, Pastoureau P, Bouet F, Bonnet J, Vanhoutte PM. Purification of guinea pig YKI40 and modulation of its secretion by cultured articular chondrocytes. J Cell Biochem 1998; 69: 414-424.

16. Millis AJ, Hoyle M, Reich E, Mann DM. Isolation and characterization of a $\mathrm{Mr}=38,000$ protein from differentiating smooth muscle cells. J Biol Chem 1985; 260: 3754-3761.

17. Lee CG, Da Silva CA, Dela Cruz CS, Ahangari F, Ma B, Kang $M-J$, et al. Role of chitin and chitinase/chitinase-like proteins in inflammation, tissue remodeling, and injury. Annu Rev Physiol 2011; 73: 479-501.

18. Prakash M, Bodas M, Prakash D, Nawani N, Khetmalas M, Mandal A, et al. Diverse pathological implications of YKL-40: answers may lie in 'outside-in' signaling. Cell Signal 2013; 25: 1567-1573.

19. Kastrup J. Can YKL-40 be a new inflammatory biomarker in cardiovascular disease? Immunobiology 2012; 217: 483-491.

20. Roslind A, Johansen JS. YKL-40: a novel marker shared by chronic inflammation and oncogenic transformation. Methods Mol Biol 2009; 511: 159-184.
21. Jockenhöfer F, Wollina U, Salva KA, Benson S, Dissemond J. The PARACELSUS score: a novel diagnostic tool for pyoderma gangrenosum. Br J Dermatol 2019; 180: 615-620.

22. Koutroubakis IE, Petinaki E, Dimoulios P, Vardas E, Roussomoustakaki M, Maniatis AN, et al. Increased serum levels of YKL-40 in patients with inflammatory bowel disease. Int J Colorectal Dis 2003; 18: 254-259.

23. Erzin Y, Uzun H, Karatas A, Celik AF. Serum YKL-40 as a marker of disease activity and stricture formation in patients with Crohn's disease. J Gastroenterol Hepatol 2008; e357-e362.

24. Bernardi D, Podswiadek M, Zaninotto M, Punzi L, Plebani M. YKL-40 as a marker of joint involvement in inflammatory bowel disease. Clin Chem 2003; 49: 1685-1688.

25. Kazakova MH, Batalov AZ, Mateva NG, Kolarov ZG, Sarafian VS. YKL-40 and cytokines - a new diagnostic constellation in rheumatoid arthritis? Folia Med (Plovdiv) 2017; 59: 37-42.

26. Hirata S, Tanaka Y. Assessment of disease activity in rheumatoid arthritis by multi-biomarker disease activity (MBDA) score. Nihon Rinsho Meneki Gakkai Kaishi 2016; 39: 37-41.

27. Peltomaa R, Paimela L, Harvey S, Helve T, Leirisalo-Repo M. Increased level of YKL-40 in sera from patients with early rheumatoid arthritis: a new marker for disease activity. Rheumatol Int 2001; 20: 192-196.

28. Kazakova M, Batalov A, Deneva T, Mateva N, Kolarov Z, Sarafian V. Relationship between sonographic parameters and YKL-40 levels in rheumatoid arthritis. Rheumatol Int 2013; 33: 341-346.

29. Zivanović S, Rackov LP, Vojvodić D, Vucetić D. Human cartilage glycoprotein 39 - biomarker of joint damage in knee osteoarthritis. Int Orthop 2009; 33: 1165-1170.

30. Brahe $\mathrm{CH}$, Dehlendorff $\mathrm{C}, \varnothing$ stergaard $\mathrm{M}$, Johansen JS, $\varnothing$ rnbjerg LM, Hørslev-Petersen K, et al. Circulating serum interleukin-6, serum chitinase-3-like protein-1, and plasma vascular endothelial growth factor are not predictive for remission and radiographic progression in patients with early rheumatoid arthritis: post-hoc explorative and validation studies based on the CIMESTRA and OPERA trials. Scand J Rheumatol 2018; 47: 259-269.

31. Salomon J, Piotrowska A, Matusiak $Ł$, Dzięgiel P, Szepietowski JC. Chitinase-3-like protein 1 (YKL-40) is expressed in lesional skin in hidradenitis suppurativa. In Vivo 2019; 33: 141-143.

32. Matusiak $\measuredangle$, Salomon J, Nowicka-Suszko D, Bieniek A, Szepietowski JC. Chitinase-3-like protein 1 (YKL-40): novel biomarker of hidradenitis suppurativa disease activity? Acta Derm Venereol 2015; 95: 736-737.

33. Ebrahim A, Mustafa AI, El-Shimi OS, Fathy MA. Serum YKL40: A novel potential link between inflammation and dyslipidemia in acne vulgaris. J Cosmet Dermatol 2020; 19: 1219-1223.

34. Salomon J, Matusiak $Ł$, Nowicka-Suszko D, Szepietowski JC. Chitinase-3-like protein 1 (YKL-40) is a biomarker of severity of joint involvement in psoriatic arthritis. Adv Dermatol Allergol 2018; 35: 485-489.

35. Boyd TA, Eastman PS, Huynh DH, Qureshi F, Sasso EH, Bolce $\mathrm{R}$, et al. Correlation of serum protein biomarkers with disease activity in psoriatic arthritis. Expert Rev Clin Immunol 2020; 16: 335-341.

36. Seo J, Ahn Y, Zheng Z, Kim BO, Choi MJ, Bang D, et al. Clinical significance of serum YKL-40 in Behçet disease. $\mathrm{Br}$ J Dermatol 2016; 174: 1337-1344.

37. Chen C, Liang Y, Zhang Z, Zhang Z, Yang Z. Relationships between increased circulating YKL-40, IL-6 and TNF-a levels and phenotypes and disease activity of primary Sjögren's syndrome. Int Immunopharmacol 2020; 88: 106878.

38. Nielsen AR, Plomgaard P, Krabbe KS, Johansen JS, Pedersen BK. IL-6, but not TNF-a, increases plasma YKL-40 in human subjects. Cytokine 2011; 55: 152-155.

39. Vind I, Johansen JS, Price PA, Munkholm P. Serum YKL-40, a potential new marker of disease activity in patients with inflammatory bowel disease. Scand J Gastroenterol 2003; 38: 599-605.

40. Salomon J, Matusiak L, Nowicka-Suszko D, Szepietowski JC. Chitinase-3-like protein 1 (YKL-40) is a new biomarker of inflammation in psoriasis. Mediators Inflamm 2017; 2017: 9538451. 